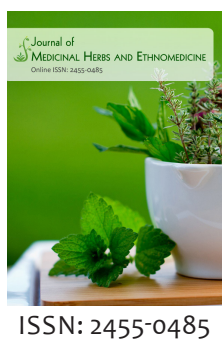

Received: November 21, 2018 Accepted: January 14, 2019 Published: January 15, 2019

*Corresponding Author: Matteo Politi

Email: matteo.politi@

takiwasi.com

\section{Phytochemical profiling of Amazonian herbal medicinal products: The role of traditional processing methods}

\author{
Matteo Politi' ${ }^{1 *}$, Giorgia Tresca', Marco Soffiato', Richer Garay Montes², \\ Fernando Mendive ${ }^{1}$ \\ 'Center for Drug Addiction Treatment and Research on Traditional Medicines - Takiwasi, Prolongación Alerta 466, \\ Tarapoto, Peru, ${ }^{2}$ Faculty of Agroindustrial Engineering, National University of San Martín, Jr. Maynas 177, Tarapoto, Peru
}

\begin{abstract}
Research on traditional processing methods used to transform plants into medicines offers a valuable arena to investigate the correct formulation of herbal products. While scientific literature on traditional herbal medicines emphasizes the relevance of the taxonomical identification of the used species, the same rigor is not applied to recording the processing methods. In other words, attention is given to the ingredients, but not to the recipes. This can not only jeopardize the quality of research on traditional herbal medicines, but also generate concerns once such medicines reach the global markets in compliance with modern manufacturing requirements, which are usually well far away from the traditional ones. In the present work, different traditional herbal medicines used in the region of San Martin, Peru, were analyzed by the means of UPLC-UV/DAD metabolite fingerprinting. Different extracts were prepared from same commercially available materials in order to test the effect of the recipes on the chemistry of the finished products. In particular, different plant parts and their status (fresh or dry), extraction solvents and decoction times were selected for metabolite comparisons between the extracts. In accord with literature data on others traditional pharmacopoeias, our results show how specific manufacturing steps can affect the chemistry of the finished products. Further efforts are necessary to evaluate the traditional herbal practices, whether understandable or not, with modern scientific approaches.
\end{abstract}

KEYWORDS: Traditional herbal medicine, mode of preparation, processing methods, herbal recipes, traditional quality standard, ethnopharmaceutics

\section{INTRODUCTION}

Due to the incorporation of modern HMPs (Herbal Medicinal Products) into current science, quality, safety, and efficacy, the three main parameters used to evaluate every category of medicine, have become an important concern also for this class of natural remedies $[1,2]$. Depending on the country-specific regulation, the definition of "Traditional" HMPs refers to the fact that such products have been used for a period of time long enough to avoid safety and efficacy controls apart from literature review (see, for instance, the European Directive 2004/24/EC), while quality remains to be measured. In any case, manufactures are obliged to comply with the general rules of the pharmaceutical sector, including those referring to Good Manufacturing Practices. Moreover, for the case of MAPs (Medicinal and Aromatic Plants), the application of Good Agricultural and Collection Practices can be also required. Both mentioned practices can strongly affect the chemistry of the finished products in terms of plant secondary metabolite content which represent the very same Active Pharmaceutical
Ingredient, a crucial parameter to verify the quality of this class of natural derivatives.

It is known that environmental, genetic, ontogenic, and morphogenetic factors can be responsible for the fluctuation in plant secondary metabolites even if in presence of cultivated pure lines of selected species. [3] Recently, the relevance of traditional wild harvesting and management methods, also in relation to the quality of raw MAPs materials has been highlighted in the Himalayan context, where serious concerns arise due to the harvest of MAPs "without taking care of traditional norms". [4] An equivalent concern in the Amazonian region refers to the psychoactive brew globally well-known with the name Ayahuasca, an admixture of two major plants, Banisteriopsis caapi and Psychotria viridis, which are traditionally collected in the wild. In fact, major fluctuation of the main bioactive alkaloids has been observed in relation to the collection sites and time of the day. [5]

The quality of the finished herbal medicines depends, however, not only on the nature of the ingredients used for the 
preparation (i.e., the plant species), but also on the processing methods applied, as well documented especially in Herbal Traditional Chinese Medicine [6]. Chinese pharmacopoeias and classical texts related to herbal Materia Medica are very rich in information concerning the so-called processing methods, which represent the necessary manufacturing steps in between the plant collection and the finished medicine. "The main purposes of processing are reducing toxicity and enhancing the effects of crude drugs. The chemical components of crude drugs and processed products differ: new components may be formed or the relative contents of certain components may change; other components may disappear or their contents may decrease".[7] Within this article, authors mention several examples of such chemical modification extracted directly from Chinese literature, including the following: "For Cortex Magnoliae Officinalis (Houpu), the contents of magnolol and honokiol increased by about $140 \%$ and $40 \%$, respectively, after stir-frying with ginger"; "Studies of the chemical changes in the constituents of Radix Euphorbiae Kansui (Cugansui, processed with vinegar) indicated that seven constituents disappeared and four new constituents were formed in the water extract of processed products while the contents of four other components decreased. In the methanol extract, two original constituents disappeared, two new constituents were produced, and the concentration of six other components increased obviously"; "The change in quantity and quality of components in volatile oils of crude Semen Myristicae (Roudoukou) and its processed product stirfried with bran were observed. The results showed that thirteen new components were found and four components disappeared in volatile oils after processing. Also, the contents of the active components methyleugenol and methylisoeugenol increased. At the same time the contents of the toxic ingredients myristicin and safrol decreased".

Proper processing methods can lead to safe and effective medicines from well-known toxic plant parts, as for the case of the Aconitum roots. [8] In this case, the content of highly toxic Aconitine and related alkaloids are strongly reduced by the application of traditional methods including soaking, decocting, steaming, and baking, as well as the co-processing with other herbal drugs (Radix Glycyrrhizae, for instance), a method which generates synergistic effects reducing the overall toxicity of the preparation. The chemical constituents of crude drugs may change dramatically during processing as for the Chinese Materia Medica cases reported above. However, apart from this well documented traditional pharmacopoeia, most of traditional knowledge related to the subject of medicinal plant processing, especially when referred to all practices whether "explicable or not" as suggested by the WHO definition of Traditional Medicine [9], remains largely unaddressed worldwide. For example, chanting "icaros" upon the finished herbal medicines is a characteristic practice of TAM (Traditional Amazonian Medicine) [10]. It is largely widespread in the Amazonia as relevant step of the traditional pharmaceutical recipes, it represents a typical example of cultural significant phenomena which still remains not explicable under the current scientific paradigm. Scientific literature reports few promising case studies measuring the so called "intention effect" on finished products: one example refers to the consumption of different chocolate samples treated and non-treated with ritual chants and shamanic melodies, followed by questionnaire-based tests to monitor the human mood in the search of "mindmatter interaction phenomenon" [11]. A second refers to the consumption of tea cups treated and non-treated with "good intentions" by Buddhist monks. [12] In both cases, however, no chemical analysis was performed on the selected samples. These kinds of intentional practices also belong to other traditional herbal cultures [13]. Thus, further studies on whole traditional processing methods are necessary in both, theoretical and practical terms, especially to provide compatibility of proper processing methods between traditional experiences and new technologies. In this context, metabolite fingerprint approaches and metabolomics are suitable techniques to investigate the proper phytochemical content which characterizes a finished herbal medicine.[14] In particular, these analyses can allow the understanding of the chemical transformations occurring during the preparation steps, therefore helping to define and standardize the correct processing methods to prepare an herbal medicine. Especially when referring to THMPs (Traditional Herbal Medicinal Products), where safety and efficacy controls are lacking because given by default due to the long history of use of such preparations, the application of traditional collecting as well as processing methods should be rigorously applied. Inconsistency of ancient and current processing methods has been already observed concerning Chinese herbal medicines, with the recommendation that "Before comparative studies are carried out, discarding the ancient experience would be inappropriate". [7] If such comparative studies are not carried out, only the strict application of traditional (i.e., long tested) collecting and processing methods can lead to obtain finished products which have been consumed historically, and that therefore can be properly considered safe and effective.

While certain traditional pharmacopoeias describe in written texts the ancient herbal formula or recipe (like for the case of the Chinese ones), in other cultural contexts such as, for instance, the African or the Amazonian one, the traditional knowledge related to the concept of Good Agricultural and Collecting Practice as well as Good Manufacturing Practice has been, and it is still being, transmitted orally. In these cases, a detailed recording of these traditional practices is urgently needed to contrast the related loss of knowledge that comes with social and cultural change.

The present work has been inspired by this kind of necessity, and allowed the recording of different mode of preparations of herbal medicines from the Peruvian Amazon in the region of San Martin. The presented results refer to the phytochemical fingerprinting analysis of different Amazonian herbal extracts performed with the aim to verify the effect of different processing methods. Commercially available MAPs and THMPs were analyzed by UPLC-UV/DAD to obtain secondary metabolite fingerprinting of crude extracts following traditional recipes and modern formulations. MAPs were acquired directly from the market and local providers, therefore voucher specimens were not recorded. The proposed scientific names presented along the text were derived from the common names used in the region and comparison with local specialized literature $[15,16]$ and 
dedicated websites (i.e. http://www.theplantlist.org). Moreover, the taxonomical identification of the MAPs here analyzed falls outside the scope of the present article, which instead aims to focus attention on the role of the processing methods on a herbal medicine at large. All MAPs here analyzed are part of the common traditional herbal knowledge in the region. They are also used routinely within the 25 years old therapeutic protocol of Takiwasi Center for the rehabilitation of drug addicted patients, based on the combination of modern psychotherapy and traditional Amazonian medicine [17-19]. In particular, Ajo Sacha (Mansoa alliacea) represents an herbal product available as a THMPs (Tincture registered by the Peruvian health authority under the national regulation DS_010_1997, manufactured by the Natural Products Laboratory of Takiwasi Center), and as a traditional remedy as well; it is mainly indicated as anti-inflammatory and anti-microbial agent, and according to Takiwasi's perspective, it is used to strengthen will power. Ushpawasha Sanango (Tabernaemontana undulata) represents a traditional remedy with a potential to reach the market as a registered THMPs; this plant is known in the region as an health tonic and used to treat rheumatism and malaria [16]. It is claimed to help metabolize personal memories of emotional nature, according to Takiwasi. Finally, Azucena (Lilium spp.) is one of the several vomiting agents which are typical therapeutic tools of TAM also used within the Takiwasi protocol, probably more difficult to integrate within the modern context as registered THMP.

\section{MATERIAL AND METHODS}

\section{Sample Preparation}

Ajo Sacha (Mansoa alliacea (Lam.) A.H. Gentry. - Bignoniaceae). Commercial hydro-ethanol extract (Tincture 20\% w/v, 80\% v/v) prepared by using dried whole roots of the plant was purchased by the Natural Product Laboratory of Takiwasi Center (batch number 108015 , identified here as Aj1). Fresh whole roots were purchased by the supplier Ampik Sacha, an association of local producers of MAPs. Root bark was obtained with the help of the traditional healers of Takiwasi Center which were peeling out manually with knifes the root bark. The fresh root bark (Aj2), the inner root (Aj3; root without the bark), and the whole root $(\mathrm{Aj} 4)$, were extracted by macerating for 3 weeks a proportion of $20 \mathrm{~g}$ of plant material in $100 \mathrm{ml}$ hydro-ethanol solution $80 \% \mathrm{v} / \mathrm{v}$ to obtain the corresponding Tinctures.

Azucena (Lilium spp. - Liliaceae). Fresh bulbs were acquired from the local market, then washed at Takiwasi Center by local healers and covered with cold water $(10 \% \mathrm{w} / \mathrm{v})$ and heated with wood fire till boiling, then left gently boiling for 8 hours. Sampling of $10 \mathrm{ml}$ were recovered every hour. Analytical results refer to samples after 2 hours (Azl) and 8 hours' (Az2) decoction.

Ushpawasha Sanango (Tabernaemontana undulata Vahl - Apocynaceae). Fresh root bark was acquired from the local market, then washed at Takiwasi Center by local healers and covered with cold water $(20 \% \mathrm{w} / \mathrm{v})$, later heated with wood fire till boiling, then left gently boiling for 8 hours. Analytical results refer to sample after 8 hours' decoction (Usl) in comparison with the Tincture (Us2) prepared by macerating for 3 weeks a proportion of $20 \mathrm{~g}$ of plant material in $100 \mathrm{ml}$ hydro-ethanol solution $80 \% \mathrm{v} / \mathrm{v}$.

\section{UPLC-UV/DAD Analyses}

UPLC-UV/DAD experiments were performed on a UHPLC Dionex Ultimate 3000, Thermo Fisher Scientific, San Jose, CA, USA, using a Column Luna ${ }^{\circledR} 5 \mu \mathrm{m} \mathrm{C18(2)} 100 \AA$, Size $150 \times 4.6 \mathrm{~mm}$, Chromeleon ${ }^{\mathrm{TM}}$ 6.8 Chromatography Data System-CDS- Software, equipped with XRS auto-sampler and UltraViolet/Diode Array Detector. $5 \mu \mathrm{L}$ of raw Tincture and aqueous extracts were injected for each sample. The flow rate was $1 \mathrm{ml} / \mathrm{min}$ with the following gradient of solvents $\mathrm{A}=\mathrm{H} 2 \mathrm{O}$ $+\mathrm{HCOOH} 0.1 \% \mathrm{v} / \mathrm{v}$ and solvents $\mathrm{B}=\mathrm{MeOH}: 0$ minutes $\mathrm{A} / \mathrm{B}$ (95/5), 10 minutes (0/100), (maintained for 2 minutes). UV/DAD spectra were recorded between 190-600 nm (chromatograms in Figure 1-4 refer to the wavelength of $254 \mathrm{~nm}$ ).

\section{RESULTS AND DISCUSSION}

\section{Plant Parts and Status Effect}

Optimization of the LC conditions was obtained on the commercially available Ajo Sacha Tinctures. Figure 1 shows the LC chromatogram of a batch prepared from dried whole roots of Ajo Sacha (Ajl), where the major classes of different natural products could be separated and partially identified by the means of the corresponding UV spectra and comparison with literature data [20-22]. In particular, between 2 and 4 min, a major phenol derivative (single $\lambda_{\text {max }}$ at ca $262.21 \mathrm{~nm}$ ) is detected at $2.63 \mathrm{~min}$. Several flavonoid-type derivatives, showing two principal $\lambda_{\text {max }}$ at ca 270 and $340 \mathrm{~nm}$, are detected between 5.50 and $7.50 \mathrm{~min}$; this is the main class of natural products detected in this extracts. Further peaks appear between 9.50 and 10.50 min showing no typical $\lambda_{\max }$ values apart from that one around $190 \mathrm{~nm}$. This data about the UV spectra, in combination with the low polarity of these molecules (high retention times), suggests that these compounds may belong to the class of Sulphur derivatives which is characteristic of this botanical genus [20].

In the region of the study and within therapeutic protocol used at Takiwasi Center, the fresh root bark of Ajo Sacha is the preferred plant part used to prepare the remedy, obtained by cold aqueous maceration of the hand squeezed plant material. This very same remedy has been also analyzed with the LC method here proposed; no significant peaks were detected probably due to the low concentration of the injected solution (data not shown). No concentration steps were performed in order to avoid any possible chemical alteration and further investigations are needed on this sample. However, metabolite fingerprinting analysis of the Ajo Sacha tinctures obtained from different parts of fresh roots was performed and the results are shown in Figure 2. The general features of the chromatograms appear similar to the above mentioned commercial Tincture Ajl, 


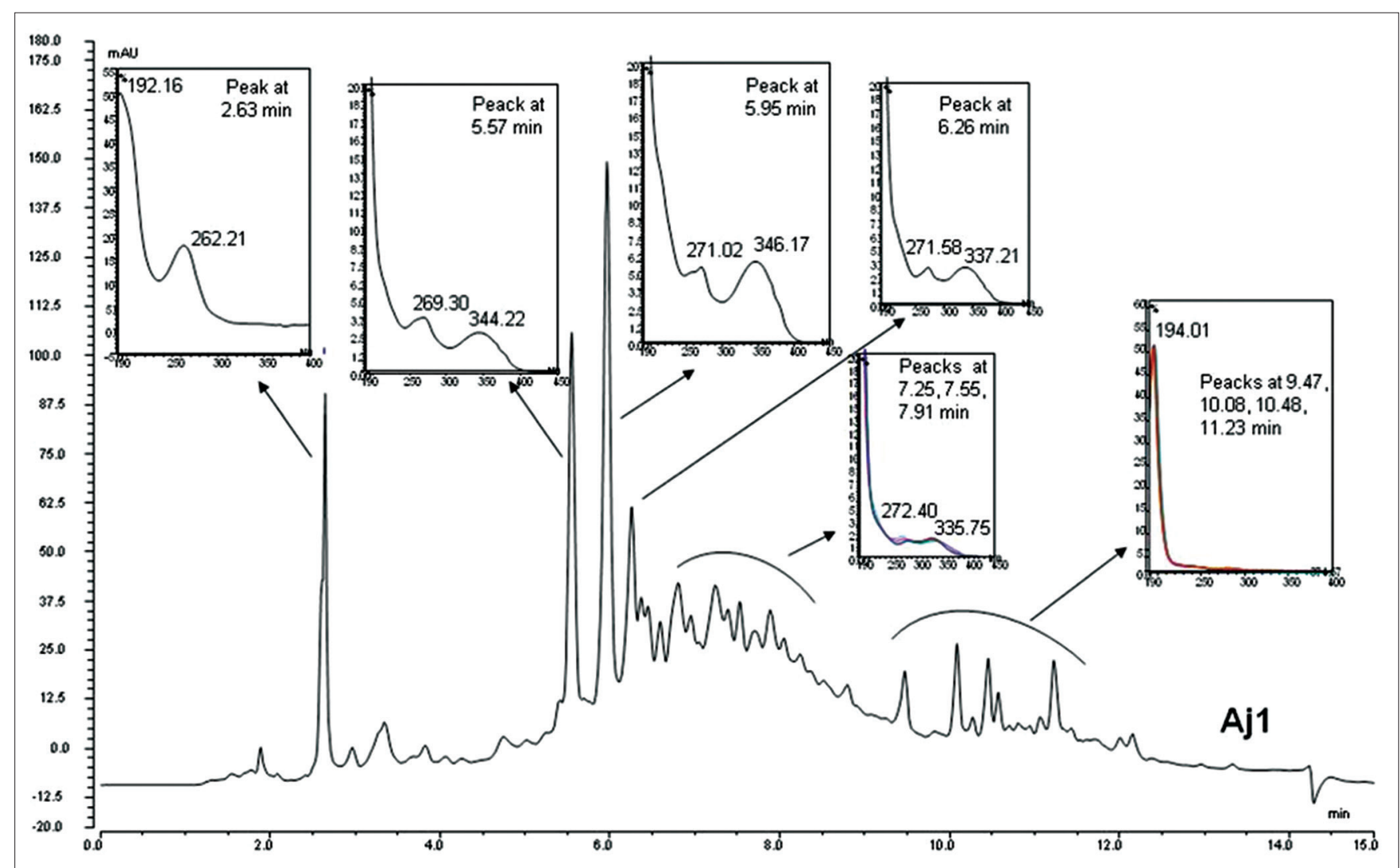

Figure 1: LC-UV chromatogram of Ajo Sacha commercial Tincture prepared from dried whole roots (Aj1), including UV spectra of major detected peaks

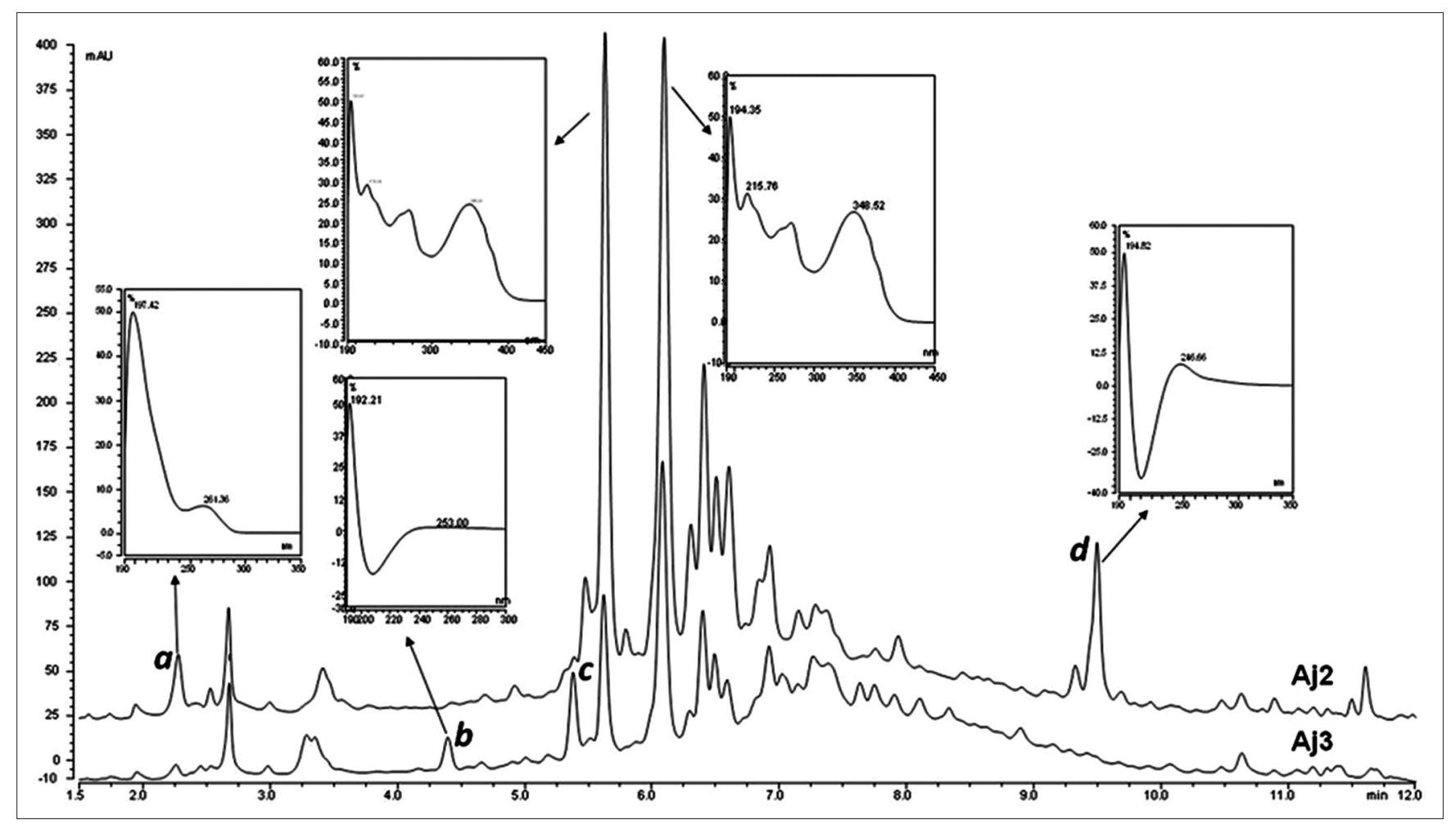

Figure 2: LC-UV chromatograms comparison of Ajo Sacha Tinctures obtained from different parts of the fresh roots including root bark (Aj2) and the inner root (Aj3; root without the bark). UV spectra of major compounds and main unshared peaks labelled a-d are presented 
flavonoid derivatives remaining the major components detected between 5.50 and $7.50 \mathrm{~min}$. However, when comparing tinctures prepared with root bark $(\mathrm{Aj} 2)$ to the ones with the inner root (Aj3; root without the bark), such flavonoid derivatives appear to be quantitatively more abundant in the root bark. These also represent the proper part of the plant used traditionally according to our ethnographic data. Moreover, labelled $a$ - $d$ peaks in Figure 2 highlight major qualitative differences between both extracts. Peaks $b$ and $c$ are present only in Tincture Aj3, which

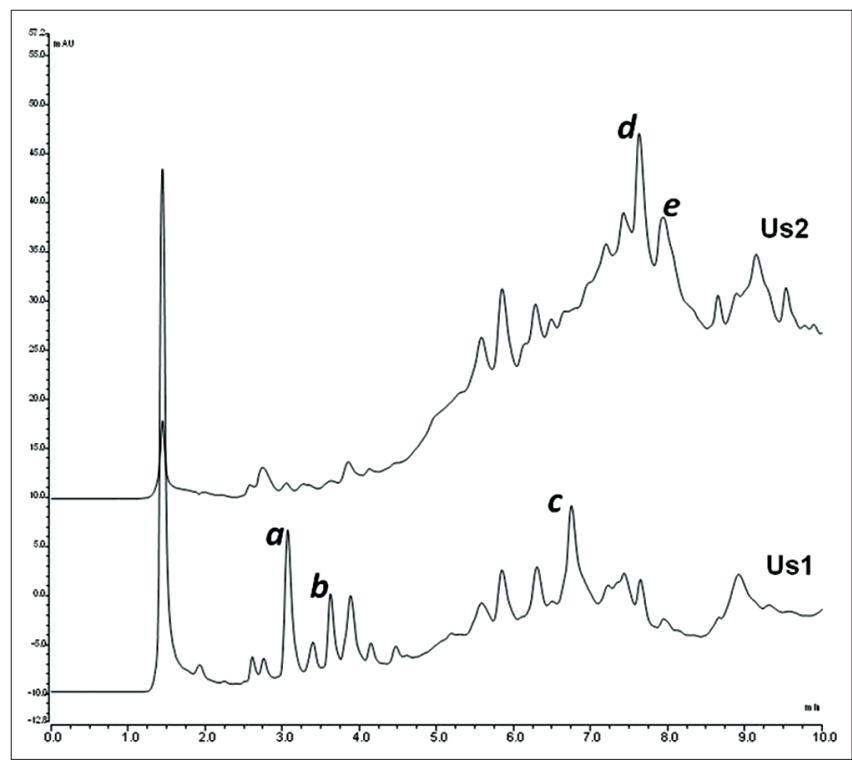

Figure 3: LC-UV chromatograms comparison of Ushpawasha Sanango Tincture (Us2) and long decoction extract (Us1). Main unshared peaks labelled $a-e$ was prepared by using the inner root, a part of the plant which is not used traditionally. Therefore, these compounds should not be present in the finished THMP prepared from Ajo Sacha. However, in preparing the same hydro-ethanol extract with the whole root (Aj4), compound $b$ especially appears to be present in the corresponding extract (data not shown). Finally, compound $d$ appears only in the root bark; it appears in the retention time region belonging to supposed Sulphur derivatives. This compound could be Allicin obtained from the precursor Alliin after peeling the roots, similarly to what happens when crashing Garlic bulbs; in fact, both compounds have been detected also in Ajo Sacha [20]. It is known that the hot air-drying process induces degradation of Allicin into further polysulfides [23, 24]. This processing step has been applied for the preparation of the commercial Tincture Ajl and could be responsible for the appearance of the peaks between 9.50 and $10.50 \mathrm{~min}$ in Figure 1.

\section{Solvent Effect}

The effect of different mediums used for the extraction of the phyto-complex, which is the plant secondary metabolite mixture characterizing the Active Pharmaceutical Ingredient of a due plant under the current scientific paradigm, has been investigated through the example of Ushpawasha Sanango herbal medicine and shown in Figure 3. In particular, the Tincture prepared as described above was compared with the traditional preparation, consisting in a long decoction as detailed in the materials and methods. The general feature of both chromatograms shows two major ranges where different peaks are detected. The first, between 2.50 and $4.50 \mathrm{~min}$, appears particularly rich in more polar derivatives among the long decoction extract (Us1), while the second, between 5 to

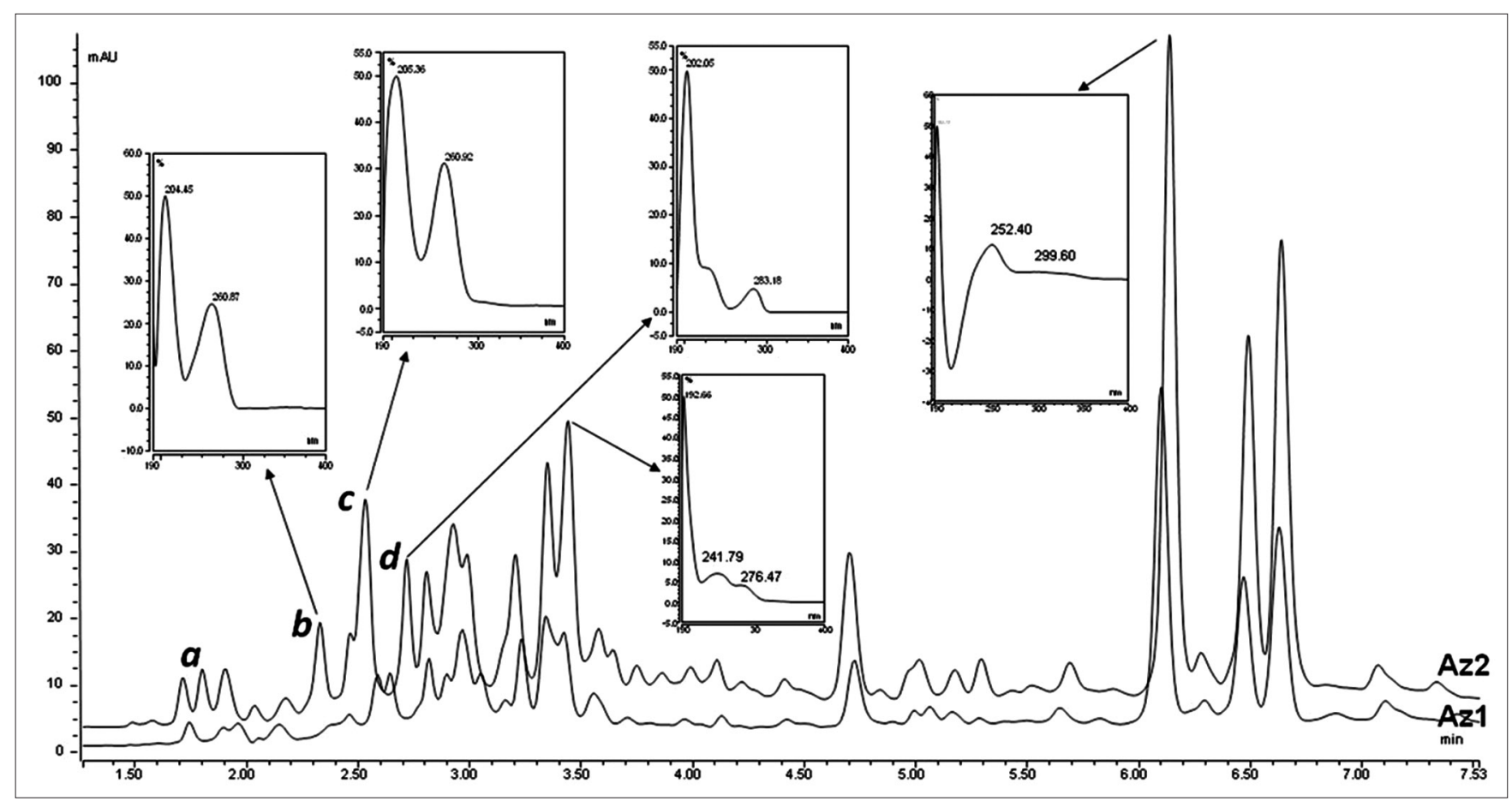

Figure 4: LC-UV chromatograms comparison of Azucena aqueous extract after 2 hours' decoction (Az1) and 8 hours' decoction (Az2). UV spectra of major compounds and main unshared peaks labelled $a-d$ are presented 
10 min, indicates a higher amount of less polar components in the hydro-ethanol extract (Us2); this is in accord with the theoretical framework of solvent polarity extraction capacity. Specific major diversity between both extracts is indicated with the unknown labelled compounds a-e. The solvent effect on the chemistry of a finished herbal product is a well-known phenomenon and appears to be rather obvious to be mentioned here. However, most of industrialized, hi-tech herbal products which reach the global market in the form of herbal medicines or food supplements are prepared by using non-traditional processing methods, including the solvents used for the extraction of the plant material. Therefore, it is still worthy to mention how a simple change like the one presented here for the case of Ushpawasha Sanango can strongly alter the chemistry of the finished product. This indicates that with the very same raw plant material it is possible to prepare different phyto-complexes and this can be both an advantage and a matter of concern. Before scaling up to commercial products based on traditional remedies, fingerprinting analysis of the traditional preparation should be carried out.

\section{Timing Effect}

Long decoction is typical to traditional preparation of Amazonian herbal medicines; even the preparation of the well-known Ayahuasca brew is based on this procedure. Recently, the variation of the major alkaloids in this beverage during the decoction time has been monitored, revealing significant variations of the bioactive compounds during the preparation [25]. Once again, this indicates that the relevance of traditional knowledge, is not limited to choosing the right plants from the environment, but is also based on the ability to process them to prepare safe and effective mixtures of natural products.

The effect of different cooking times has been registered here for the case of Azucena decoctions. Chromatograms in Figure 4 refer to samples after 2 hours (Azl) and 8 hours' decoction (Az2). Overall quantitative difference between both extracts is observed, indicating that the Az2 extract is richer in the shared compounds compare to Azl. Moreover, qualitative changes are also detected as indicated with the labelled $a-d$ peaks. These unknown compounds are extracted from the plant material or formed during the prolonged cooking process. These results clearly indicate that, when studying traditional herbal medicines especially within scientific disciplines such as ethnobotany or ethnopharmacology, the detailed description of the preparation modes is necessary. Both extracts here analyzed could be generally registered as "decoction", although the exact timing of this recipes may represent a key factor to prepare the correct mixture of natural products from this plant material in order obtain the expected pharmacological effect.

\section{CONCLUSIONS}

The present work shows the effects of certain processing methods on the chemistry of finished Amazonian herbal medicines. In particular, the exact choice of the plant part and status was investigated through the analysis of Ajo Sacha samples; the use of different extraction solvents was evaluated by analyzing Ushpawasha Sanango extracts; the cooking time effect was monitored for the case of Azucena preparation. Quantitative and qualitative variations were detected for all the analyzed cases. This clearly points at the need for more in depth investigation into such practices, especially when developing modern herbal formulations from traditional medicines without relying on safety and efficacy tests. Processing methods are part of the overall know-how on herbal medicines and should be considered as the traditional equivalent of Good Manufacturing Practice. Therefore, can be considered as a set of strict regulations which have to be followed in order to obtain the desired herbal medicines.

In the present study, the evaluation of a limited number of manufacturing steps belonging to the concept of processing method was performed, although further specific and more complex pathways surely merit more attention. Moreover, as for the case of other traditional pharmacopoeias, the Amazonian one includes a huge number of examples of complex herbal formulas. Further studies could be very promising not only in terms of discovery of potential synergistic effects [26], but also as a key to identify new substances which can be generated during the manufacturing process of such traditional remedies. The generation of new molecules during the cooking of multiingredient preparation is well known within the subject of food chemistry. In this case, a specific sub-discipline has been created specifically for the study of this kind of chemical reactions, namely "Molecular Gastronomy"[27]. Under the concept of "Food processing" it is claimed that "only with the preparation of the raw ingredients in the kitchen and then combining them and cooking them in the appropriate manner that the true quality of a plate of food is developed" [28]; this should be considered also in the field of "herbal processing" in order to obtain "true quality" medicines. New bioactive molecules can even be discovered through the chemical analysis of very common ingredients such as extra virgin olive oil, if the analysis takes place on a food preparation. [29] The very same could occur if medicinal plants would be studied within their well established context of use. We therefore believe that an equivalent subdiscipline of pharmaceutical science should be dedicated to the study of the traditional processing methods used for the preparations of medicines, whether understandable or not under the current scientific paradigm. This could be properly named "Ethnopharmaceutics".

\section{REFERENCES}

1. Mandal, S.C. and M. Mandal, Quality, Safety, and Efficacy of Herbal Products Through Regulatory Harmonization. Drug Information Journal, 2011. 45: 45-53.

2. Govindaraghavan, S. and N. Sucher, Quality assessment of medicinal herbs and their extracts: Criteria and prerequisites for consistent safety and efficacy of herbal medicines. Epilepsy \& Behavior, 2015. 52(Pt B): 363-371.

3. Verma, N. and S. Shukla, Impact of various factors responsible for fluctuation in plantsecondary metabolites. Journal of Applied Research on Medicinal and Aromatic Plants, 2015. 2(4): 105-113.

4. Sharma, N. and C. Kala, Harvesting and management of medicinal and aromatic plants in the Himalaya. Journal of Applied Research 
on Medicinal and Aromatic Plants, 2017. in press(https://doi. org/10.1016/j.jarmap.2017.09.003).

5. Callaway, J., G. Brito, and E. Neves, Journal of Psychoactive Drugs. Phytochemical Analyses of Banisteriopsis Caapi and Psychotria Viridis, 2005. 37(2): 145-150.

6. Sheridan, H., et al., Traditional Chinese herbal medicine preparation: Invoking the butterfly effect, in The Art and Science of Traditional Medicine Part 3: The Global Impact of Traditional Medicine. 2015, (6262) Sponsored supplement to Science. p. S53-S85.

7. Zhao, Z., et al., A unique issue in the standardization of Chinese materia medica: processing. Planta Med, 2010. 76(17): 1975-86.

8. Liu, S., et al., A review of traditional and current methods used to potentially reduce toxicity of Aconitum roots in Traditional Chinese Medicine. Journal of Ethnopharmacology, 2017. 207: 237-250.

9. Zhang, Q., http://who.int/medicines/areas/traditional/definitions/en/. 2000(28/06/2017)

10. Callicott, C., Interspecies communication in the Western Amazon: Music as a form of conversation between plants and people. European Journal of Ecopsychology, 2013. 4: 32-43.

11. Radin, D., G. Hayssen, and J. Walsh, Effects of intentionally enhanced chocolate on mood. Explore-the Journal of Science and Healing, 2007. 3(5): 485-492.

12. Shiah, Y. and D. Radin, Metaphysics of the tea ceremony: a randomized trial investigating the roles of intention and belief on mood while drinking tea. Explore-the Journal of Science and Healing, 2013. 9(6): 355-360.

13. Politi, M. and L. Pisani, Traditional pharmaceutical recipes from a metabolomics perspective: Interviews with traditional healers in Mali and comparison with modern herbal medicines Advancement in Medicinal Plant Research, 2014 2(1): 1-6.

14. Cao, H., et al., The application of metabolomics in traditional Chinese medicine opens up a dialogue between Chinese and Western medicine. Phytotherapy Ressearch, 2014. 29(2): 159-166

15. Brack Egg, A., Diccionario Enciclopedico de Plantas Utiles del Peru. 1999, Cuzco: CBC - Centro de Estudio Regionales Andinos "Bartolomé de Las Casas"

16. Sanz-Biset, J. and S. Cannigueral, Plant use in the medicinal practices known as "strict diets" in Chazuta valley (Peruvian Amazon). Journal of Ethnopharmacology, 2011. 137 271- 288.
17. Politi, M. and F. Friso, Amazonian Medicinal Plants Botanical Garden of Takiwasi Center in Peru; a case report of 25 years' hands-on experience. Horticulture International Journal, 2018. 2(3): 69-71.

18. Politi, M., F. Friso, and J. Mabit, Plant based assisted therapy for the treatment of substance use disorders - part 1. The case of Takiwasi Center and other similar experiences. Revista Cultura y Droga, 2018. 23 (26): 99-126.

19. Berlowitz, I., et al., Conceptions and practices of an integrative treatment for substance use disorders involving Amazonian medicine: traditional healers' perspectives. Revista Brasileira de Psiquiatria, 2018. 40(2): 200-209.

20. Bichara Zoghbi, M., J. Oliveira, and G. Skelding Pinheiro Guilhon, The genus Mansoa (Bignoniaceae): a source of organosulfur compounds. Brazilian Journal of Pharmacognosy, 2009. 19(3): 795-804.

21. Patel, I., et al., Phytochemical studies on Mansoa alliacea (Lam.). International Journal of Advances in Pharmaceutical Research, 2013. 4(6): $1823-1828$.

22. Pires, F., et al., Qualitative and quantitative analysis of the phenolic content of Connarus var. angustifolius, Cecropia obtusa, Cecropia palmata and Mansoa alliacea based on HPLC-DAD and UHPLC-ESIMS/MS. Revista Brasileira de Farmacognosia, 2017. 27 426-433.

23. Ratti, C., et al., Drying of Garlic (Allium sativum) and Its Effect on Allicin Retention. Drying Technology, 2007. 25: 349-356.

24. Papu, S., et al., Effect of Drying Characteristics of Garlic-A Review. J Food Process Technol 2014. 5(4).

25. Cardoso Santos, M., S. Navickiene, and A. Gaujac, Determination of Tryptamines and $\beta$-Carbolines in Ayahuasca Beverage Consumed During Brazilian Religious Ceremonies. Journal of AOAC International, 2017. 100(3): 820-824

26. Wagner, H. and G. Ulrich-Merzenich, Synergy research: approaching a new generation of phytopharmaceuticals. Phytomedicine, 2009. 16(2-3): 97-110.

27. This, H., Molecular gastronomy. Angew Chem Int Ed Engl., 2002. 41(1): 83-88.

28. Barham, P., et al., Molecular Gastronomy: A New Emerging Scientific Discipline. Chem. Rev., 2010. 110: 2313-2365.

29. Sacchi, R., et al., Extra virgin olive oil: from composition to "molecular gastronomy". Cancer Treat Res., 2014. 159: 325-338. 